\title{
Neue Horizonte der Pflegewissenschaft
}

\section{Graz zum 3. Mal Gastgeber der European Doctoral Conference in Nursing Science}

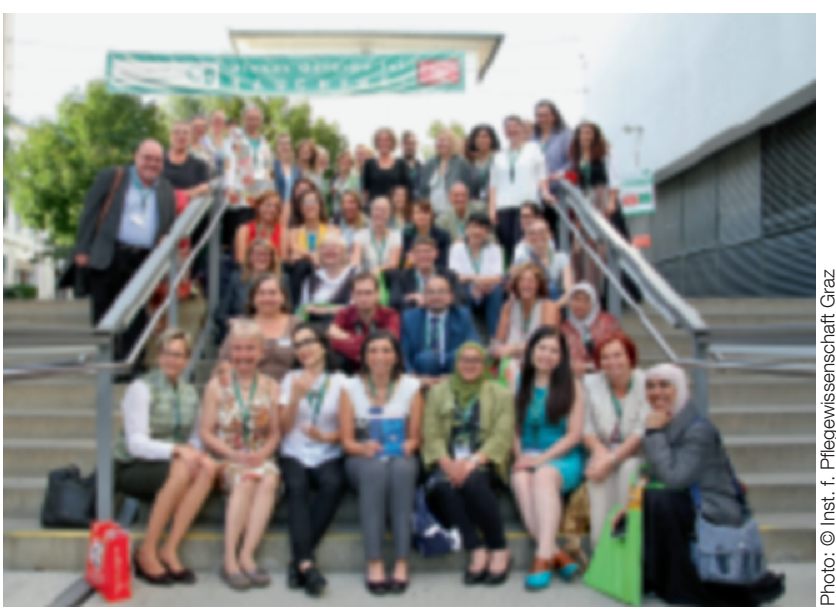

Der Erfahrungs- und Erkenntnisaustausch von Doktoranden, Dozenten und Professoren

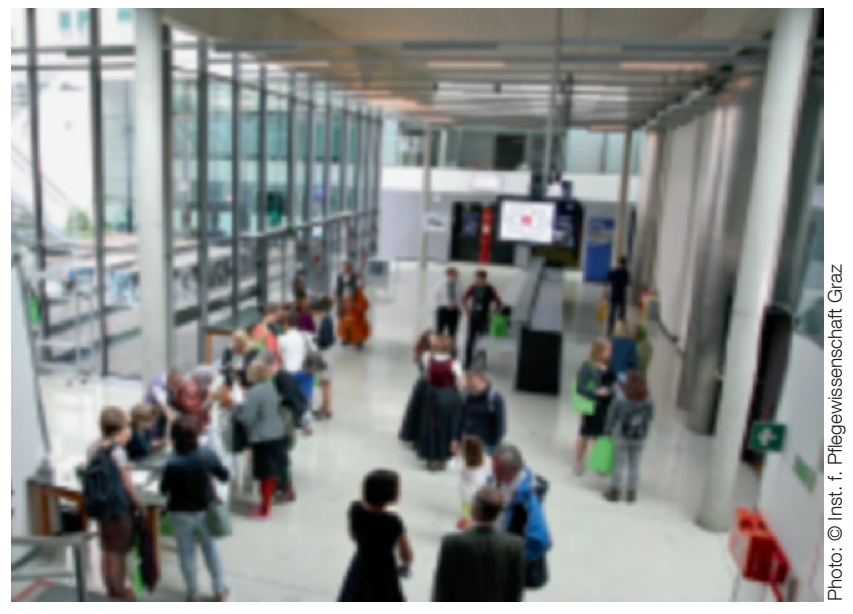

.. steht bei der EDCNS im Mittelpunkt.
"Exploring horizons in Nursing Science“ lautete das Motto der Europäischen Doktorandenkonferenz für Pflegewissenschaft (European Doctoral Conference in Nursing Science - EDCNS), die zum dritten Mal an der Medizinischen Universität Graz stattfand. Ziel der Konferenz: Erfahrungen im Bereich der Pflegeforschung untereinander auszutauschen und Erkenntnisse über derzeit in der Pflegewissenschaft in Europa beforschte Themen zu erlangen. Dazu kamen Mitte September knapp 80 Doktoranden, Dozenten und Professoren aus 22 unterschiedlichen Ländern nach Graz.

International einmalig, ist der Fokus der EDCNS sowohl bei der Organisation als auch während der Konferenz ausschließlich auf die Doktoranden und ihre Forschungsprojekte gerichtet. Dieser Aspekt wurde unter anderem auch während der feierlichen Eröffnungszeremonie durch Landesrat Mag. Christopher Drexler, Rektor Univ.-Prof. Dr. Josef Smolle, Dekan Univ.-Prof. Dr. Peter Holzer und der Institutsvorständin und Gründerin der EDCNS, Univ.-Prof. Dr. Christa Lohrmann aufgegriffen. Das vielfältige Programm der Konferenz setzte sich aus Vorträgen, Posterpräsentationen und Workshops zu den unterschiedlichsten pflegewissenschaftlichen Themen zusammen.

„Diese Konferenz eröffnete den Teilnehmern verschiedenste Möglichkeiten. Einerseits konnten Kollegen aus ganz
Europa getroffen und Netzwerke aufgebaut werden. Andererseits konnten Forschungsprojekte mit pflegewissenschaftlichem Fokus präsentiert und diskutiert werden, sowie Erfahrungen hinsichtlich Pflegeforschung geteilt und erörtert werden. Workshops mit internationalen Referenten boten Möglichkeiten einer themenspezifischen Vertiefung“, erklären die Organisatorinnen der diesjährigen Konferenz, Manuela Mandl, Silvia Bauer, Sandra Schüssler, Nursiswati und Daniela Schoberer vom Institut für Pflegewissenschaft der Medizinischen Universität Graz.

Quelle: Presseaussendung Institut für Pflegewissenschaft der Medizinischen Universität Graz Informationen: http://bit.ly/1P38dxF

\section{IMPRESSUM}

Herausgeber und Verleger: Springer-Verlag GmbH, Professional Media, Prinz-Eugen-Str. 8-10, Postfach 11, 1040 Wien, Austria, Tel.: 01/330 24 15, Fax: 01/33024 26 Internet: www.springer.com, www.SpringerMedizin.at Geschäftsführung: Dr. Alois Sillaber, Petrus J.W. Hendriks, Joachim Krieger Leitung: Dr. Alois Sillaber Leitung Journale und Redaktionen Medizin: Gabriele Hollinek Redaktion: Verantwortlicher Redakteur: Verena Kienast Mitarbeiter dieser Ausgabe: Dipl. Tzt. Elise Haidenthaller Redaktionssekretariat: Susanna Hinterberger Produktion: Dorothea Wolinski Leitung Verkauf Medizin: Robert Seiwald Anzeigen: Gabriele Popernitsch; Es gilt die Anzeigenpreisliste 2015. Erscheinungsweise: 10× jährlich Abonnement: Michaela Bolli; Bezugspreis pro Jahr: für Institutionen EUR 109,- zuzüglich MwSt. und Versandkosten Bezugsbedingungen: Das Abonnement für Einzelbezieher gilt mit Bezug des ersten Heftes jeweils fur ein Jahr mit der in der Preisliste für einen vollen Jahrgang angegebenen Anzahl von Ausgaben. Abbestellungen innerhalb dieser Laufzeit konnen nicht entgegengenommen werden. Das Verogrt: Win Zert:

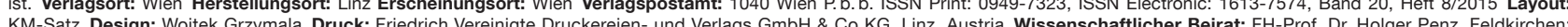
KM-Satz Design: Wojtek Grzymala Druck: Friedrich Vereinigte Druckereien- und Verlags GmbH \& Co KG, Linz, Austria Wissenschaftlicher Beirat: FH-Prof. Dr. Holger Penz, Feldkirchen; Univ.-Prof. Dr. Christa Lohrmann, Graz, Univ-Piof. Dr. Christa Them, Hall. T., Univ. Prof. Hanna Mayer, Wien, FH-Prof. Dr. Roswitha Engel, Wien, Unv.-Prof. Dr. Jurgen Osterbrink, Salzburg; Dr. Elisabeth Rappold, Wien; Maria Jesse, Wien; Charlotte Staudinger, Wien. Alle namentlich gekennzeichneten Beitrage spiegeln nicht unbedingt die Meinung der Redaktion wider. Diese Beitrage fallen somit in den personichen Verantwortungsbereich des Verfassers. Die Redaktion übernimmt keine Haftung für unaufgefordert eingesandte Manuskripte. Mt "Sonderbericht" oder "Advertorial“" gekennzeichnete Seiten sind entgeltliche Einschaltungen nach $\$ 26$ Mediengesetz. Allgemeiner Teil/Rechtliche Hinweise für Autoren Die Autorin/der Autor erklärt, dass ihr/sein Manuskript in dieser Form bislang nicht anderweitig veröffentlicht oder zur Veröffentlichung eingereicht wurde. Die Autorin/der Autor überträgt mit der Ubergabe des fertigen Manuskripts und der Veroffentlichung in der Fachzeitschrift die notwendigen Nutzungsrechte zur Vervielfaltigung und Verbreitung an den Verlag, insbesondere das Recht der Nutzung zu gewerblichen Zwecken durch Druck, Nachdruck, Verbreitung in elektronischer Form oder andere Verfahren und Medien durch Springer Science + Business Media. Beiträge, die in PROCARE erscheinen, können
auch in der Springer-Zeitschrift Heilberufe veröffentlicht werden. Die Autorin/der Autor holt, falls notwendig, die Nutzungsrechte an Texten und Bildern Dritter vor Übergabe des fertigen Manuauch in der Springer-Zeitschrift Heilberufe veröffentlicht werden. Die Autorin/der Autor holt, falls notwendig, die Nutzungsrechte an Texten und Bildern Dritter vor Ubergabe des fertigen Manu-
skripts ein, eventuelle Ansprüche Dritter sind somit geklärt. Hinweise zur Verwertung: Die Zeitschrift sowie alle in ihr enthaltenen einzelnen Beiträge und Abbildungen sind urheberrechtlich skripts ein, eventuelle Ansprüche Dritter sind somit geklärt. Hinweise zur Verwertung: Die Zeitschrift sowie alle in ihr enthaltenen einzelnen Beiträge und Abbildungen sind urheberrechtlich
geschützt. Jede Verwertung, auch auszugsweise, die nicht ausdrücklich vom Urheberrechtsgesetz zugelassen ist, bedarf der vorherigen schriftlichen Zustimmung des Verlages. Das gilt insbegeschützt. Jede Verwertung, auch auszugsweise, die nicht ausdrücklich vom Urheberrechtsgesetz zugelassen ist, bedarf der vorherigen schriftlichen Zustimmung des Verlages. Das gilt insbeSondere für Vervielfältigungen, Bearbeitungen, Ubersetzungen, Mikroverfilmungen und die Verarbeitung in elektronischen Systemen. Produkthaftung: Die Wiedergabe von Gebrauchsnamen, Handelsnamen, Warenbezeichnungen usw. in dieser Zeitschrift berechtigt auch ohne besondere Kennzeichnung nicht zu der Annahme, dass solche Namen im Sinne der Warenzeichen- und
Markenschutz-Gesetzgebung als frei zu betrachten wären und daher von jedermann benutzt werden dürften. Angaben über Dosierungsanweisungen und Applikationsformen sind anhand anMarkenschutz-Gesetzgebung als frei zu betrachten waren und daher von jedermann benutzt werden dürtten. Angaben über Dosierungsanweisungen und Applikationsformen sind anhand anWien. Springer ist Teil von Springer Science + Business Media. Coverbild: @ monkeybusinessimages / Getty Images / thinkstock 